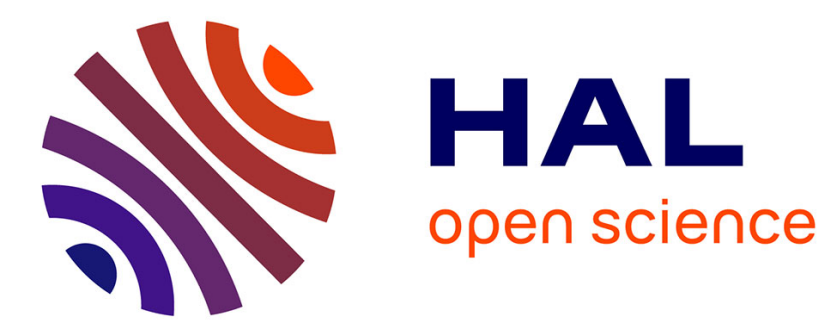

\title{
Small-time local stabilization for a Korteweg-de Vries equation
}

Shengquan Xiang

\section{To cite this version:}

Shengquan Xiang. Small-time local stabilization for a Korteweg-de Vries equation. Systems and Control Letters, 2018, Volume 111, pp.Pages 64-69. hal-01723178

\section{HAL Id: hal-01723178 \\ https://hal.science/hal-01723178}

Submitted on 5 Mar 2018

HAL is a multi-disciplinary open access archive for the deposit and dissemination of scientific research documents, whether they are published or not. The documents may come from teaching and research institutions in France or abroad, or from public or private research centers.
L'archive ouverte pluridisciplinaire HAL, est destinée au dépôt et à la diffusion de documents scientifiques de niveau recherche, publiés ou non, émanant des établissements d'enseignement et de recherche français ou étrangers, des laboratoires publics ou privés. 


\title{
Small-time local stabilization for a Korteweg-de Vries equation
}

\author{
Shengquan Xiang ${ }^{1}$
}

\begin{abstract}
This paper focuses on the (local) small-time stabilization of a Korteweg-de Vries equation on bounded interval, thanks to a time-varying Dirichlet feedback law on the left boundary. Recently, backstepping approach has been successfully used to prove the null controllability of the corresponding linearized system, instead of Carleman inequalities. We use the "adding an integrator" technique to gain regularity on boundary control term which clears the difficulty from getting stabilization in small-time.
\end{abstract}

Keywords: Korteweg-de Vries, backstepping, small-time stabilization, adding an integrator. American Subject Classification: 35Q53, 34H05, 35P10.

\section{Introduction}

We consider the stabilization problem of the Kortewegde Vries equation

$$
y_{t}+y_{x}+y_{x x x}+y y_{x}=0
$$

posed on a bounded domain $[0, L]$. This system requires three boundary conditions including both left and right end-point (see [26], the system fails to be well-posed when all boundary conditions are given at only one end-point), among which the most studied case is

$$
y(t, 0), y(t, L), y_{x}(t, L)
$$

When there is only one control term $y_{x}$, i.e. $y(t, 0)=$ $y(t, L)=0$, the phenomenon becomes quite mysterious: starting from the linearized $\mathrm{KdV}$ equation, Lionel Rosier [23] found that the system is controllable if and only if the length of the interval satisfies

$$
L \notin \mathcal{N}:=\left\{2 \pi \sqrt{\frac{l^{2}+l k+k^{2}}{3}} ; l, k \in \mathbb{N}^{*}\right\} .
$$

It allows us to decompose the $L^{2}(0, L)$ space into the controllable state and the uncontrollable state (for the linearized KdV equation). To get the controllability of the $\mathrm{KdV}$ equations, "Power Series Expansion" method was introduced in $[1,3,13]$, which turned out to be a classical example of getting controllability by using nonlinear terms. The stabilization problem is even more interesting,

\footnotetext{
${ }^{1}$ Université Pierre et Marie Curie-Paris 6, UMR 7598 Laboratoire Jacques-Louis Lions, 75005 Paris, France. ETH Zürich, Institute for Theoretical Studies, 8092 Zürich, Switzerland. ETH Zürich, Forschungsinstitut für Mathematik, 8092 Zürich, Switzerland. Email: shengquan.xiang@ens.fr. SX was supported by LIASFMA and ANR Project Finite4SoS (ANR 15-CE23-0007).
}

as we need to investigate a closed-loop system which involves more difficulties (even for the well-posedness). Several works $[5,6,15,18,22,25]$ have been made in this special KdV control system, here we refer [18] where the authors used the nonlinear term (and also uncontrollable part) to find a time-varying feedback law which stabilize the system exponentially. As we can see, in this model we really used the nonlinear term, by "fixing" the uncontrollable part, to reach the goal of controlling and stabilizing. However, at the same time, the results are only local and the stabilization is only exponential. To get the same results in global sense, is a challenging and interesting problem, since one may need to find the other techniques for the nonlinear term.

In this paper, we will focus on the control acting on $y(t, 0)$, with $y(t, L)=y_{x}(t, L)=0$. This system has an advantage of being locally controllable, see [2, 19, 24] for discussions on this system. From the stabilization point of view, the final aim should be (local) small-time stabilization, especially by means of time-varying feedback laws (inspired by [8] for the finite dimensional case). Recently in [16], Jean-Michel Coron and Hoai-Minh Nguyen made a first step, they used a piece-wise backstepping control to get the null controllability and the semi-global smalltime stabilization for the heat equation. Here we refer to $[12,14,20,21]$ for the history, explanation and development of backstepping method. As the backstepping method has already been used for a rapid stabilization for this KdV system, one may naturally expect the small-time stabilization. In the recent paper [26], the author used this technique to give a new proof of null controllability of the linearized $\mathrm{KdV}$ equation. There came a difficulty of lacking regularity on the control term $y(t, 0)$, where a priori a $H^{1 / 3}$ regularity on $y(t, 0)$ is needed, but one can hardly ensure the feedback to be more than $C^{0}$ with respect to time.

A technique called "adding an integrator" is going to 
solve our problem. Usually used to avoid the offset in the stabilization problem, this technique also has the advantage of gaining regularity. Indeed, if we "add" another term, $a(t)$, as

$$
y(t, 0)=a(t), \quad a_{t}(t)=u(t),
$$

where $u(t)$ is the control, then we will have $a(t) \in H^{1}(0, T)$ if $u(t) \in L^{2}(0, T)$. One can see the paper of Jean-Michel Coron [10], where this method is used for the stabilization of Euler equations. Let us also point out that the control system with the additional integral term has controllability and stabilizability properties which are related but may be different than the ones of the initial control system; see, in particular [11, Proposition 3.30 and Section 12.5] and [17] for finite dimensional control systems.

We consider in this paper the following system:

$$
\begin{gathered}
y_{t}+y_{x}+y_{x x x}+y y_{x}=0, \\
y(t, L)=y_{x}(t, L)=0, \\
y(t, 0)=a(t), \\
a_{t}(t)+y_{x x}(t, 0)+\frac{1}{2} a(t)+\frac{1}{3} a^{2}(t)=u(t),
\end{gathered}
$$

in the interval $[0,1]$ (we only consider only the case when $L=1$ to simplify the notations). We notice the extra $y_{x x}(t, 0)$ term in (1.8). It naturally comes from (1.7) and helps to ensure the well-posedness of our new system. As for terms $(1 / 2) a$ and $(1 / 3) a^{2}$, which could be put in the control term, we let them here to make the dissipative nature visible. It is a control system where the state is $(y(x), a)$, but with only one control $u$. Let us set

$$
V:=L^{2}(0,1) \times \mathbb{R} \text { and }\|(y, a)\|_{V}^{2}:=\|y\|_{L^{2}(0,1)}^{2}+a^{2} .
$$

Then easy calculations show that the "flow" of system (1.5)-(1.8) satisfies:

$$
\begin{aligned}
\frac{d}{d t}\|(y, a)\|_{V}^{2} & =2\left\langle y_{t}, y\right\rangle_{L^{2}}+2 a_{t} a \\
& =2\left\langle-y_{x}-y_{x x x}-y y_{x}, y\right\rangle_{L^{2}}+2 a_{t} a \\
& =a^{2}+\frac{2}{3} a^{3}-y_{x}(0)^{2}+2 a y_{x x}(0)+2 a_{t} a \\
& \leqslant 2 a u .
\end{aligned}
$$

In order to get the well-posedness of the nonlinear system, we may need some smoothing effects. We first consider the linearized system of (1.5)-(1.8). By multiplying $x y$ the linearized part of (1.5), we get the Kato smoothing effect $y \in L^{2}\left(0, T ; H^{1}(0,1)\right)$. This together with (1.10) and some fixed point argument, allows us to get the well-posedness of the nonlinear system (1.5)-(1.8) in the transposition sense (with initial data $\left(y_{0}, a_{0}\right) \in V$ and control $u(t) \in L^{1}(0, T)$ ), the solution being in

$$
\left(C^{0}\left([0, T] ; L^{2}(0,1)\right) \cap L^{2}\left(0, T ; H^{1}(0,1)\right)\right) \times C^{0}([0, T] ; \mathbb{R}) .
$$

Here, we are not going to reconstruct the whole theory of transposition solutions, which is already well explained in the book [11] (one can also see similar cases in $[16,18]$ ). Based on the method introduced in [16] and the estimates given in [26], we are able to stabilize system (1.5)-(1.8) in small time. More precisely, for every $T>0$, we will construct time-varying feedback laws $U(t ; y, a): \mathbb{R} \times L^{2}(0,1) \times$ $\mathbb{R} \mapsto \mathbb{R}$, satisfying the following three properties:

$\left(\mathcal{P}_{1}\right)$ The feedback law $U$ is $T$-periodic with respect to time:

$$
U(t ; y, a)=U(T+t ; y, a)
$$

$\left(\mathcal{P}_{2}\right)$ There exists an increasing sequence $\left\{t_{n}\right\}$ of real numbers such that

$$
\begin{gathered}
t_{0}=0, \\
\lim _{n \rightarrow \infty} t_{n}=T,
\end{gathered}
$$

$\mathrm{U}$ is of class $C^{1}$ in $\left[t_{n}, t_{n+1}\right) \times L^{2}(0,1) \times \mathbb{R}$.

$\left(\mathcal{P}_{3}\right)$ The feedback law $U$ vanishes on $\mathbb{R} \times\{0\} \times\{0\}$. There exists a continuous function $M:[0, T) \rightarrow[0,+\infty)$ such that

$$
\begin{gathered}
\left|U\left(t ; y_{1}, a_{1}\right)\right|-\left|U\left(t ; y_{2}, a_{2}\right)\right| \\
\leqslant M(t)\left(\left\|y_{1}-y_{2}\right\|_{L^{2}}+\left|a_{1}-a_{2}\right|\right),
\end{gathered}
$$

for $\forall t \in[0, T)$.

$\left(\mathcal{P}_{4}\right)$ For all $(t ; y, a) \in \mathbb{R} \times L^{2}(0,1) \times \mathbb{R}$, we have

$$
|U(t ; y, a)|<1
$$

$\left(\mathcal{P}_{5}\right)\|(y, a)\|_{V} \geqslant 1 \Rightarrow U(t ; y, a)=0$, for all $t \in \mathbb{R}$.

From now on, let us consider the Cauchy problem of the closed-loop system (1.5)-(1.8)

$$
\left\{\begin{array}{l}
y_{t}+y_{x}+y_{x x x}+y y_{x}=0, \\
y(t, 1)=y_{x}(t, 1)=0, \\
y(t, 0)=a(t), \\
a_{t}+y_{x x}(0)+\frac{1}{2} a+\frac{1}{3} a^{2}=u, \\
y(s, x)=y_{0}, \\
a(s)=a_{0}, \\
u:=U(t ; y, a)
\end{array}\right.
$$

with $(t, x) \in(s,+\infty) \times(0,1)$. For this Cauchy problem, from properties $\left(\mathcal{P}_{1}\right)-\left(\mathcal{P}_{3}\right)$ we have the existence and uniqueness of solution in small-time. A solution $\left(y_{1}, a_{1}\right)$ : $\left[s, \tau_{1}\right) \rightarrow V$ to the Cauchy problem is maximal, if there is no solution $\left(y_{2}, a_{2}\right):\left[s, \tau_{2}\right) \rightarrow V$ such that $\tau_{2}>\tau_{1}$ and $\left(y_{1}, a_{1}\right)=\left(y_{2}, a_{2}\right)$ in $\left[s, \tau_{1}\right]$. From the uniqueness of solution, let us denote $\Phi\left(t, s ; y_{0}, a_{0}\right)$ with $t \in\left[s, \iota\left(s ; y_{0}, a_{0}\right)\right]$ the unique maximal solution with initial data $\left(y_{0}, a_{0}\right)$, we will call this solution the flow of the Cauchy system (1.18). Properties $\left(\mathcal{P}_{4}\right)-\left(\mathcal{P}_{5}\right)$ let every maximal solution to be defined on $[s,+\infty)$, i.e. $\iota\left(s ; y_{0}, a_{0}\right)=+\infty$.

The main purpose of this paper is to prove the following theorem: 
Theorem 1. Let $T>0$. There exists $\varepsilon>0$ and a timevarying feedback law $U(t ; y, a)$ satisfying properties $\left(\mathcal{P}_{1}\right)$ $\left(\mathcal{P}_{5}\right)$ such that following properties hold:

(i) $\iota\left(s ; y_{0}, a_{0}\right)=+\infty$, for every $\left(s ; y_{0}, a_{0}\right) \in \mathbb{R} \times V$.

(ii) $\Phi\left(t+2 T, t ; y_{0}, a_{0}\right)=0$, if $\left\|\left(y_{0}, a_{0}\right)\right\|_{V} \leqslant \varepsilon$.

(iii) (Uniform stability property) For $\forall \delta>0, \exists \eta>0$ such that

$$
\left(\left\|\left(y_{0}, a_{0}\right)\right\|_{V} \leqslant \eta\right) \Rightarrow\left(\left\|\Phi\left(t, t^{\prime} ; y_{0}, a_{0}\right)\right\|_{V} \leqslant \delta, \forall t \geqslant t^{\prime}\right)
$$

This paper is organized as follows. In Section 2, we give a stationary feedback law $F_{\lambda}$ which can locally exponentially stabilize the system with decay rate $\lambda$. Section 3 contains the construction of the time-varying feedback law, which leads to the local small-time stabilization that we will prove in Section 4.

\section{Rapid stabilization}

This section is based on the rapid stabilization of a $\mathrm{KdV}$ system proved in [2] and estimate given in [26]. Let us start from the linearized system

$$
\left\{\begin{array}{l}
y_{t}+y_{x}+y_{x x x}=0 \\
y(t, 1)=y_{x}(t, 1)=0 \\
y(t, 0)=u(t)
\end{array}\right.
$$

It is proved in [2] that for any given positive $\lambda$, there is a kernel $k_{\lambda}$ defined in the triangle $\mathcal{T}:=\{(x, v): x \in$ $(0,1), v \in(x, 1)\}$ such that if we perform the transformation $\Pi_{\lambda}: L^{2}(0,1) \longrightarrow L^{2}(0,1)$

$$
z(x)=\Pi_{\lambda}(y(x)):=y(x)-\int_{x}^{1} k_{\lambda}(x, v) y(v) d v,
$$

then the solution $y$ of system (2.2) with feedback law

$$
u(t):=\int_{0}^{1} k_{\lambda}(0, v) y(t, v) d v
$$

is mapped to the solution $z$ of the system

$$
\left\{\begin{array}{l}
z_{t}+z_{x}+z_{x x x}+\lambda z=0 \\
z(t, 1)=z_{x}(t, 1)=0 \\
z(t, 0)=0
\end{array}\right.
$$

Therefore we have the exponential stabilization:

$$
\|z(t)\|_{L^{2}(0,1)} \leqslant e^{-\lambda t}\|z(0)\|_{L^{2}(0,1)}
$$

hence exponentially decay for the solution $y(t, \cdot)$ thanks to the invertibility of the transformation $\Pi_{\lambda}$.

As for the kernel, the following result is given in [2]:

\section{Lemma 1.}

(1) The kernel $k_{\lambda}$ satisfies equation

$$
\begin{cases}k_{x x x}+k_{v v v}+k_{x}+k_{v}+\lambda k=0 & \text { in } \mathcal{T}, \\ k(x, 1)=0 & \text { in }[0,1] \\ k(x, x)=0 & \text { in }[0,1] \\ k_{x}(x, x)=\frac{\lambda}{3}(1-x) & \text { in }[0,1] .\end{cases}
$$

(2) The inverse of transformation $\Pi_{\lambda}, \Pi_{\lambda}^{-1}$, is given by

$$
y(x)=\Pi_{\lambda}^{-1}(z(x)):=z(x)+\int_{x}^{1} l_{\lambda}(x, v) z(v) d v .
$$

And the kernel $l_{\lambda}$ satisfies

$$
\begin{cases}l_{x x x}+l_{v}+l_{x}+l_{v}-\lambda l=0 & \text { in } \mathcal{T}, \\ l(x, 1)=0 & \text { in }[0,1], \\ l(x, x)=0 & \text { in }[0,1], \\ l_{x}(x, x)=\frac{\lambda}{3}(1-x) & \text { in }[0,1] .\end{cases}
$$

Later, in [26], more information on $k_{\lambda}$ is given:

Lemma 2. The equation (2.6) has a unique solution. There exists a constant $C_{1}$, which is independent of $\lambda>1$, such that

$$
\left\|k_{\lambda}\right\|_{C^{3}(\mathcal{T})} \leqslant e^{C_{1} \sqrt{\lambda}} \text {, and }\left\|l_{\lambda}\right\|_{C^{3}(\mathcal{T})} \leqslant e^{C_{1} \sqrt{\lambda}}
$$

Remark 1. In [26], the estimate is only given for the $C^{1}$ norm, but one can easily get similar $C^{3}$ estimates by using the same method. As stated in [26], it will be a challenging and interesting problem to know whether the right hand side of (2.9) can be replaced by $\exp \left(C \lambda^{1 / 3}\right)$.

Now, we consider the linearized system of (1.18):

$$
\left\{\begin{array}{l}
y_{t}+y_{x}+y_{x x x}=0 \\
y(t, 1)=y_{x}(t, 1)=0 \\
y(t, 0)=a(t) \\
a_{t}+y_{x x}(0)+\frac{1}{2} a=u
\end{array}\right.
$$

We look for a transformation $\Xi_{\lambda}$ with $(z, b)=\Xi_{\lambda}((y, a))$ of the form:

$$
\left\{\begin{array}{l}
z:=\Pi_{\lambda}(y), \\
b:=a+F_{\lambda}(y),
\end{array}\right.
$$

and a feedback law of the form

$$
u(t):=K_{\lambda}(y)+L_{\lambda} a .
$$

We want $(z, b)$ to satisfy the following target system:

$$
\left\{\begin{array}{l}
z_{t}+z_{x}+z_{x x x}+\lambda z=0 \\
z(t, 1)=z_{x}(t, 1)=0 \\
z(t, 0)=b(t) \\
b_{t}+z_{x x}(0)+\frac{1}{2} b+\mu b=w,
\end{array}\right.
$$


with $\mu$ and $w$ to be chosen later.

Actually, performing the same calculation as in $[2$, page 1690] with (2.6), we get

$$
z_{t}+z_{x}+z_{x x x}+\lambda z=0 .
$$

Besides, we have

$$
\begin{gathered}
z(t, 1)=y(t, 1)=0, \\
z_{x}(t, 1)=y_{x}(t, 1)+k_{\lambda}(1,1) y(t, 1)=0, \\
z(t, 0)=a-\int_{0}^{1} k_{\lambda}(0, v) y(t, v) d v .
\end{gathered}
$$

Hence, $F_{\lambda}(y)$ should be

$$
F_{\lambda}(y):=-\int_{0}^{1} k_{\lambda}(0, v) y(v) d v .
$$

At last, let us calculate $w$ :

$$
\begin{aligned}
w= & b_{t}+z_{x x}(0)+\frac{1}{2} b+\mu b \\
= & \left(a+F_{\lambda}(y)\right)_{t}+\left(\mu+\frac{1}{2}\right)\left(a+F_{\lambda}(y)\right)+y_{x x}(0) \\
& -\left(\int_{x}^{1} k_{\lambda}(x, v) y(v) d v\right)_{x x}(0) \\
= & \left(a_{t}+y_{x x}(0)+\frac{1}{2} a\right)+\left(F_{\lambda}(y)\right)_{t}+\frac{1}{2} F_{\lambda}(y) \\
& -\left(\int_{0}^{1} k_{\lambda, x x}(0, v) y(v) d v-k_{\lambda, x}(0,0) a\right)+\mu\left(a+F_{\lambda}(y)\right)
\end{aligned}
$$

Since

$$
\begin{aligned}
\left(F_{\lambda}(y)\right)_{t}= & -\int_{0}^{1} k_{\lambda}(0, v) y_{t}(t, v) d v \\
= & \int_{0}^{1} k_{\lambda}(0, v)\left(y_{x}(t, v)+y_{x x x}(t, v)\right) d v \\
= & -\int_{0}^{1} k_{\lambda, v}(0, v) y(t, v) d v+\left.k_{\lambda}(0, v) y(t, v)\right|_{0} ^{1} \\
& -\int_{0}^{1} k_{\lambda, v v v}(0, v) y(t, v) d v+\left.k_{\lambda}(0, v) y_{x x}(t, v)\right|_{0} ^{1} \\
& -\left.k_{\lambda, v}(0, v) y_{x}(t, v)\right|_{0} ^{1}+\left.k_{\lambda, v v}(0, v) y(t, v)\right|_{0} ^{1} \\
= & -\int_{0}^{1}\left(k_{\lambda, v}(0, v)+k_{\lambda, v v v}(0, v)\right) y(t, v) d v \\
& +k_{\lambda, v}(0,0) y_{x}(t, 0)-k_{\lambda, v v}(0,0) a
\end{aligned}
$$

from (2.10)-(2.20) we get

$$
\begin{gathered}
w=u-\int_{0}^{1}\left(k_{\lambda, v}+k_{\lambda, v v v}+\frac{1}{2} k_{\lambda}\right. \\
\left.\quad+\mu k_{\lambda}+k_{\lambda, x x}\right)(0, v) y(t, v) d v \\
-\left(-k_{\lambda, x}(0,0)+k_{\lambda, v v}(0,0)-\mu\right) a+k_{\lambda, v}(0,0) y_{x}(t, 0) \\
=u-\left(-k_{\lambda, x}(0,0)+k_{\lambda, v v}(0,0)-\mu\right) a+k_{\lambda, v}(0,0) z_{x}(t, 0) \\
-\int_{0}^{1}\left(k_{\lambda, v}+k_{\lambda, v v v}+\frac{1}{2} k_{\lambda}\right. \\
\left.\quad+\mu k_{\lambda}+k_{\lambda, x x}+\frac{\lambda}{3} k_{\lambda, x}\right)(0, v) y(t, v) d v
\end{gathered}
$$

where we used the fact that

$$
z_{x}(t, 0)-y_{x}(t, 0)=-\int_{0}^{1} k_{\lambda, x}(0, v) y(v) d v
$$

Hence we define the feedback, $u(t)=K_{\lambda}(y)+L_{\lambda} a$, by

$$
\left\{\begin{aligned}
K_{\lambda}(y):= & \int_{0}^{1}\left(k_{\lambda, v}+k_{\lambda, v v v}+\frac{1}{2} k_{\lambda}\right. \\
& \left.+\mu k_{\lambda}+k_{\lambda, x x}+\frac{\lambda}{3} k_{\lambda, x}\right)(0, v) y(v) d v, \\
L_{\lambda}:=- & k_{\lambda, x}(0,0)+k_{\lambda, v v}(0,0)-\mu,
\end{aligned}\right.
$$

which leads to

$$
w=k_{\lambda, v}(0,0) z_{x}(t, 0)=-\frac{\lambda}{3} z_{x}(t, 0) .
$$

Let us choose

$$
\mu:=\lambda^{2}+\lambda
$$

From (2.13), (2.23) and (2.24), we get

$$
\begin{aligned}
\frac{d}{d t}\|(z, b)\|_{V}^{2} & =-z_{x}(0)^{2}-2 \lambda\|z\|_{L^{2}}^{2}-2\left(\lambda^{2}+\lambda\right) b^{2}+2 w b \\
& \leqslant-2 \lambda\|(z, b)\|_{V}^{2}
\end{aligned}
$$

which leads to the (global) exponential decay with rate $\lambda$ to the target system (2.13). In order to get exponential decay to the system (2.10), we need to point that both $\Xi_{\lambda}, \Xi_{\lambda}^{-1}: V \rightarrow V:$

$$
\begin{aligned}
\Xi_{\lambda}:\left(\begin{array}{c}
y \\
a
\end{array}\right) & \longrightarrow\left(\begin{array}{cc}
\Pi_{\lambda} & 0 \\
F_{\lambda} & 1
\end{array}\right)\left(\begin{array}{l}
y \\
a
\end{array}\right) \\
\Xi_{\lambda}^{-1}:\left(\begin{array}{c}
z \\
b
\end{array}\right) & \longrightarrow\left(\begin{array}{cc}
\Pi_{\lambda}^{-1} & 0 \\
-F_{\lambda} \Pi_{\lambda}^{-1} & 1
\end{array}\right)\left(\begin{array}{c}
z \\
b
\end{array}\right),
\end{aligned}
$$

are bounded.

From (2.2), (2.18), (2.22) and Lemma 2, there exists $C_{2}$ independent of $\lambda>1$ such that following estimates on the norm of operators hold

$$
\begin{gathered}
\left|L_{\lambda}\right| \leqslant e^{C_{2} \sqrt{\lambda}}, \quad\left|K_{\lambda}\right| \leqslant e^{C_{2} \sqrt{\lambda}},\left|F_{\lambda}\right| \leqslant e^{C_{2} \sqrt{\lambda}} \\
\left|\Pi_{\lambda}\right| \leqslant e^{C_{2} \sqrt{\lambda}} \text { and }\left|\Pi_{\lambda}^{-1}\right| \leqslant e^{C_{2} \sqrt{\lambda}} .
\end{gathered}
$$

Hence

$$
\left|\Xi_{\lambda}\right| \leqslant 2 e^{2 C_{2} \sqrt{\lambda}} \text { and }\left|\Xi_{\lambda}^{-1}\right| \leqslant 2 e^{2 C_{2} \sqrt{\lambda}}
$$

Let us consider now the stability of nonlinear system (1.18) with feedback law $u$ given by (2.12) and (2.22). Suppose that $(y, a)(t)$ is a solution of (1.18) with (2.12), then $(z, b):=\Xi_{\lambda}(y, a)$ satisfies

$$
\begin{gathered}
z_{t}(t, x)+z_{x}(t, x)+z_{x x x}(t, x)+\lambda z(t, x) \\
=-\left(z(t, x)+\int_{x}^{1} l_{\lambda}(x, v) z(t, v) d v\right) \\
\cdot\left(z_{x}(t, x)+\int_{x}^{1} l_{\lambda, x}(x, v) z(t, v) d v\right) \\
-\frac{1}{2} \int_{x}^{1} k_{\lambda, v}(x, v)\left(\Pi_{\lambda}^{-1} z\right)^{2}(t, v) d v=I,
\end{gathered}
$$




$$
\begin{aligned}
& z(t, 1)=0, z_{x}(t, 1)=0, z(t, 0)=b \\
& b_{t}+z_{x x}(0)+\frac{1}{2} b+\mu b+\frac{\lambda}{3} z_{x}(t, 0) \\
= & -\frac{1}{3} a^{2}-\frac{1}{2} \int_{0}^{1} k_{\lambda, v}(0, v) y^{2}(t, v) d v \\
= & -\frac{1}{3}\left(F_{\lambda} \Pi_{\lambda}^{-1} z-b\right)^{2}-\frac{1}{2} \int_{0}^{1} k_{\lambda, v}(0, v)\left(\Pi_{\lambda}^{-1} z\right)^{2}(t, v) d v \\
= & J
\end{aligned}
$$

Hence, together with (2.24), the flow $(z, b)$ satisfy

$$
\begin{aligned}
\frac{d}{d t}\|(z, b)\|_{V}^{2}= & -z_{x}(0)^{2}-2 \lambda\|z\|_{L^{2}}^{2}-2\left(\lambda^{2}+\lambda\right) b^{2}-\frac{2 \lambda}{3} z_{x}(0) b \\
& +2\langle z, I\rangle_{L^{2}}+2 b J \\
\leqslant & -2 \lambda\|(z, b)\|_{V}^{2}+2\langle z, I\rangle_{L^{2}}+2 b J
\end{aligned}
$$

Performing the same calculation as in [2, page 1692], we get

$$
\left|2\langle z, I\rangle_{L^{2}}\right|+2|b J| \leqslant e^{C_{3} \sqrt{\lambda}}\|(z, b)\|_{V}^{3},
$$

with $C_{3}>3 C_{2}$ independent of $\lambda>1$.

Hence if the initial state $\left(z_{0}, b_{0}\right)$ satisfies

$$
\left\|\left(z_{0}, b_{0}\right)\right\|_{V} \leqslant e^{-C_{3} \sqrt{\lambda}}\left(i . e .\left\|\left(y_{0}, a_{0}\right)\right\|_{V} \leqslant e^{-2 C_{3} \sqrt{\lambda}}\right),
$$

the solution $(z, b)$ will have the exponential decay

$$
\|(z, b)(t)\|_{V} \leqslant e^{-\frac{\lambda}{2} t}\left\|\left(z_{0}, b_{0}\right)\right\|_{V} .
$$

\section{Control design}

This section is devoted to the construction of the feedback law. As what is done in [26], we will find a piecewise continuous feedback on time $[0, T)$ such that properties $\left(\mathcal{P}_{2}\right)-\left(\mathcal{P}_{5}\right)$ holds. Actually, once we find this feedback on $[0, T)$, we can prolong it periodically to get a feedback law fulfills $\left(\mathcal{P}_{1}\right)-\left(\mathcal{P}_{5}\right)$. Since the feedback law (2.12) given in Section 2 is Lipschitz in $V$, it is not difficult to design such piecewise feedback laws.

The difficult part is the choice of $\left\{\lambda_{n}\right\}$ (increasing positive numbers that tend to infinity) and $\left\{t_{n}\right\}$ (increasing numbers with $\mathbf{t}_{\mathbf{0}}=\mathbf{0}$ that tend to $\mathbf{T}$ as $\mathbf{n}$ tends to infinity), such that Theorem 1 holds.

For any piece $\left[t_{n}, t_{n+1}\right)$, let us define

$$
\left.u(t ; y, a)\right|_{t \in\left[t_{n}, t_{n+1}\right)}:=\varphi_{\lambda_{n}}\left(\|(y, a)\|_{V}\right)\left(K_{\lambda_{n}}(y)+L_{\lambda_{n}} a\right),
$$

where $\varphi_{\lambda_{n}}:=\mathbb{R}^{+} \rightarrow \mathbb{R}^{+}$is given by

$$
\left\{\begin{array}{c}
1, \quad \text { if } x \in\left[0, e^{-C_{2} \sqrt{\lambda_{n}}} / 5\right], \\
2-5 e^{C_{2} \sqrt{\lambda_{n}}} x, \quad \text { if } x \in\left[e^{-C_{2} \sqrt{\lambda_{n}}} / 5,2 e^{-C_{2} \sqrt{\lambda_{n}}} / 5\right] \\
0, \quad \text { if } x \in\left[2 e^{-C_{2} \sqrt{\lambda_{n}}} / 5,+\infty\right) .
\end{array}\right.
$$

Actually, one can easily verify that properties $\left(\mathcal{P}_{2}\right)-\left(\mathcal{P}_{5}\right)$ hold for proper choice of $\left\{t_{n}\right\}$. Let us directly choose

$$
\begin{gathered}
t_{n}:=0, \lambda_{n}:=0 \text { for } n<n_{0}:=1+\left[\frac{2}{\sqrt{T}}\right], \\
t_{n}:=T-1 / n^{2}, \lambda_{n}:=2 n^{8}, \text { for } n \geqslant n_{0}:=1+\left[\frac{2}{\sqrt{T}}\right] .
\end{gathered}
$$

We prove Theorem 1 with this feedback law in the next section.

\section{Small-time stabilization}

The proof of Theorem 1 is divided into three parts:

(1) The solution exists in arbitrary time.

(2) There exists $\varepsilon>0$ such that, $\Phi\left(T, 0 ; y_{0}, a_{0}\right)=0$, if $\left\|\left(y_{0}, a_{0}\right)\right\|_{V} \leqslant \varepsilon$.

(3) Uniform stability property, see (1.19).

In fact, (1) equals to (i), (3) equals to (iii), and (2)-(3) imply (ii).

Let us start by (1). By classical fixed point argument, for every $R>0$, we know the existence of $T_{R}$ such that for every initial state $\|(y, a)\|_{V}<R$, the solution exists on $\left(0, T_{R}\right)$. We only need to verify that the solution will never blow-up. Following the simple calculation in (1.10) with the help of $\left(\mathcal{P}_{4}\right)-\left(\mathcal{P}_{5}\right)$, we can control the $V$-norm of the solution in arbitrary time. As the time-varying feedback law is bounded at every time except $t=T$, we also need to prove that for $\forall s \in[0, T)$, following limit

$$
\lim _{t \rightarrow T^{-}} \Phi\left(t, s ; y_{0}, a_{0}\right)
$$

exists. This can be proved by using the same method given in $[16$, page 22$]$. We omit it here.

The next and the most important step is to prove (2). On time $\left[t_{n}, t_{n+1}\right)$, the feedback $u$ is given by

$$
\varphi_{\lambda_{n}}\left(\|(y, a)\|_{V}\right)\left(K_{\lambda_{n}}(y)+L_{\lambda_{n}} a\right) .
$$

We observe that if $\varphi_{\lambda_{n}} \neq 1$, the exponential decay no longer holds. The idea is to prove that

$$
\left.\varphi_{\lambda_{n}}(\|(y, a)(t)) \|_{V}\right) \equiv 1, \text { for } t \in\left[t_{n}, t_{n+1}\right)
$$

which is equivalent to have that

$$
\|(y, a)(t)) \|_{V} \leqslant e^{-C_{2} \sqrt{\lambda_{n}} / 5} \text {, for } t \in\left[t_{n}, t_{n+1}\right) .
$$

As we have seen in Section 2, in order to get exponential stabilization of our nonlinear system (1.18), the following condition on the "initial state"

$$
\left\|(y, a)\left(t_{n}\right)\right\|_{V} \leqslant e^{-2 C_{3} \sqrt{\lambda_{n}}}
$$

is sufficient. One can simply verify the following lemma:

Lemma 3. For every $n \geqslant 1$. For every

$$
\left\|(y, a)\left(t_{n}\right)\right\|_{V} \leqslant e^{-4 C_{3} \sqrt{\lambda_{n}}},
$$

conditions (4.2)-(4.3) hold for $t \in\left[t_{n}, t_{n+1}\right)$. 
If both (4.2) and (4.4) are fulfilled, the solution $(y, a)(t)$ is controlled by the following estimate:

For $t \in\left[0, T-1 / n_{0}^{2}\right]$, we have $\|(y, a)(t)\|_{V} \leqslant\left\|\left(y_{0}, a_{0}\right)\right\|_{V}$. For $t \in\left[t_{n}, t_{n+1}\right)$ with $n \geqslant n_{0}$, we have

$$
\begin{aligned}
& \|(y, a)(t)\|_{V} /\left\|\left(y_{0}, a_{0}\right)\right\|_{V} \\
\leqslant & \left|\Xi_{\lambda_{n}} \| \Xi_{\lambda_{n}}^{-1}\right| \prod_{k=n_{0}}^{n-1}\left(\left|\Xi_{\lambda_{k}}\right|\left|\Xi_{\lambda_{k}}^{-1}\right| e^{-\left(t_{k+1}-t_{k}\right) \lambda_{k} / 2}\right) \\
\leqslant & \left(\prod_{k=n_{0}}^{n-1} e^{-c k^{5}}\right)\left(\prod_{k=n_{0}}^{n} e^{5 C_{2} k^{4}}\right) .
\end{aligned}
$$

In order to ensure the conditions (4.2) and (4.4), and to get the stabilization to 0 on time $T$, we only need to find $\varepsilon>0$ such that

$$
\varepsilon\left(\prod_{k=n_{0}}^{n-1} e^{-c k^{5}}\right)\left(\prod_{k=n_{0}}^{n} e^{5 C_{2} k^{4}}\right) \leqslant e^{-4\left(C_{2}+C_{3}\right) n^{4}}
$$

for all $n \geqslant n_{0}$. Such $\varepsilon$ obviously exists.

At last, it remains to prove (3), the uniform stability property. On the one hand, observe from (1.10) and $\left(\mathcal{P}_{4}\right)$ that, for $\forall \delta_{0}>0$, there exists $T_{0} \in[0, T)$ such that

$$
\begin{aligned}
& \left(\left\|\left(y_{0}, a_{0}\right)\right\|_{V} \leqslant \delta_{0} / 2, t_{0} \in\left[T_{0}, T\right)\right) \\
\Rightarrow & \left(\left\|\Phi\left(t, t_{0} ; y_{0}, a_{0}\right)\right\|_{V} \leqslant \delta_{0}, \forall t \in\left[t_{0}, T\right)\right) .
\end{aligned}
$$

On the other hand, from $\left(\mathcal{P}_{3}\right)$ we can find a $M$ such that

$$
u(t ; y, a) \leqslant M\|(y, a)\|_{V}, \text { for } t \in\left[0, T_{0}\right],
$$

which concludes the existence of $C$ such that

$$
\left\|\Phi\left(t, s ; y_{0}, a_{0}\right)\right\|_{V} \leqslant C\left\|\left(y_{0}, a_{0}\right)\right\|_{V}, \forall 0 \leqslant s \leqslant t \leqslant T_{0} .
$$

Estimates (4.6) and (4.8) together with (2) give the uniform stability property (3), which completes the proof.

Remark 2. As we have seen, the main idea is to use the "kernel" (linear part), which forces our results to be local. From the controllability point of view, one can use the return method to get global control results (even in small time), see [4, 7, 9]. From local stabilization to some global result, there still exists a big gap, especially for small-time.

Acknowledgments. The author would like to thank Jean-Michel Coron for having attracted his attention to this problem, for his constant support, and for fruitful discussions. He also thanks Amaury Hayat, Peipei Shang and Christophe Zhang for discussions on this problem.

\section{References}

[1] Eduardo Cerpa. Exact controllability of a nonlinear Kortewegde Vries equation on a critical spatial domain. SIAM J. Control Optim., 46(3):877-899 (electronic), 2007.

[2] Eduardo Cerpa and Jean-Michel Coron. Rapid stabilization for a Korteweg-de Vries equation from the left Dirichlet boundary condition. IEEE Trans. Automat. Control, 58(7):1688-1695, 2013.
[3] Eduardo Cerpa and Emmanuelle Crépeau. Boundary controllability for the nonlinear Korteweg-de Vries equation on any critical domain. Ann. Inst. H. Poincaré Anal. Non Linéaire, 26(2):457-475, 2009.

[4] Marianne Chapouly. Global controllability of a nonlinear Korteweg-de Vries equation. Commun. Contemp. Math., 11(3):495-521, 2009.

[5] Jixun Chu, Jean-Michel Coron, and Peipei Shang. A note on the Gevrey property of the linear operator for KdV equation. Preprint, 2017.

[6] Jixun Chu, Jean-Michel Coron, and Peipei Shang. Asymptotic stability of a nonlinear Korteweg-de Vries equation with critical lengths. J. Differential Equations, 259(8):4045-4085, 2015.

[7] Jean-Michel Coron. Global asymptotic stabilization for controllable systems without drift. Math. Control Signals Systems, $5(3): 295-312,1992$

[8] Jean-Michel Coron. On the stabilization in finite time of locally controllable systems by means of continuous time-varying feedback law. SIAM J. Control Optim., 33(3):804-833, 1995.

[9] Jean-Michel Coron. On the controllability of 2-D incompressible perfect fluids. J. Math. Pures Appl. (9), 75(2):155-188, 1996.

[10] Jean-Michel Coron. On the null asymptotic stabilization of the two-dimensional incompressible Euler equations in a simply connected domain. SIAM J. Control Optim., 37(6):1874-1896, 1999.

[11] Jean-Michel Coron. Control and nonlinearity, volume 136 of Mathematical Surveys and Monographs. American Mathematical Society, Providence, RI, 2007.

[12] Jean-Michel Coron. Stabilization of control systems and nonlinearities. In Proceedings of the 8th International Congress on Industrial and Applied Mathematics, pages 17-40. Higher Ed. Press, Beijing, 2015.

[13] Jean-Michel Coron and Emmanuelle Crépeau. Exact boundary controllability of a nonlinear KdV equation with critical lengths. J. Eur. Math. Soc. (JEMS), 6(3):367-398, 2004.

[14] Jean-Michel Coron and Brigitte d'Andréa Novel. Stabilization of a rotating body beam without damping. IEEE Trans. Automat. Control, 43(5):608-618, 1998.

[15] Jean-Michel Coron and Qi Lü. Local rapid stabilization for a Korteweg-de Vries equation with a Neumann boundary control on the right. J. Math. Pures Appl. (9), 102(6):1080-1120, 2014.

16] Jean-Michel Coron and Hoai-Minh Nguyen. Null controllability and finite time stabilization for the heat equations with variable coefficients in space in one dimension via backstepping approach. Arch. Ration. Mech. Anal., to appear, 2017.

[17] Jean-Michel Coron and Laurent Praly. Adding an integrator for the stabilization problem. Systems Control Lett., 17(2):89-104, 1991.

[18] Jean-Michel Coron, Ivonne Rivas, and Shengquan Xiang. Local exponential stabilization for a class of Korteweg-de Vries equations with time-varying feedback law. Anal. PDE, to appear, 2017.

[19] Olivier Glass and Sergio Guerrero. Some exact controllability results for the linear $\mathrm{KdV}$ equation and uniform controllability in the zero-dispersion limit. Asymptot. Anal., 60(1-2):61-100, 2008.

[20] Miroslav Krstic and Andrey Smyshlyaev. Boundary control of PDEs, volume 16 of Advances in Design and Control. Society for Industrial and Applied Mathematics (SIAM), Philadelphia, PA, 2008. A course on backstepping designs.

[21] Wei-Jiu Liu and Miroslav Krstić. Backstepping boundary control of Burgers' equation with actuator dynamics. Systems Control Lett., 41(4):291-303, 2000.

[22] Gustavo Alberto Perla Menzala, C. F. Vasconcellos, and Enrique Zuazua. Stabilization of the Korteweg-de Vries Equation with localized damping. Q. Appl. Math., LX(1):111-129, 2002.

[23] Lionel Rosier. Exact boundary controllability for the Kortewegde Vries equation on a bounded domain. ESAIM Control Optim. Calc. Var., 2:33-55 (electronic), 1997.

[24] Lionel Rosier. Control of the surface of a fluid by a wavemaker. ESAIM Control Optim. Calc. Var., 10(3):346-380 (electronic), 
2004.

[25] Shuxia Tang, Jixun Chu, Peipei Shang, and Jean-Michel Coron. Local asymptotic stability of a KdV system with a twodimensional center manifold. Adv. Nonlinear Anal., 2016.

[26] Shengquan Xiang. Null controllability of a linearized Kortewegde Vries equation by backstepping approach. Preprint, 2016. 